\title{
Метод матриц рассеяния для расчета спонтанной излучательной рекомбинации в структурах с цилиндрической симметрией
}

\author{
(C) В.В. Николаев ${ }^{1}$, К.А. Иванов ${ }^{2,3}$, К.М. Морозов ${ }^{2,3}$, А.В. Белоновский ${ }^{2}$ \\ ${ }^{1}$ Физико-технический институт им. А.Ф. Иоффее Российской академии наук, \\ 194021 Санкт-Петербург, Россия \\ 2 Университет ИТМО, \\ 197101 Санкт-Петербург, Россия \\ ${ }^{3}$ Санкт-Петербургский Академический университет им. Ж.И. Алфёрова Российской академии наук, \\ 194021 Санкт-Петербург, Россия \\ E-mail: Valentin.Nikolaev@mail.ioffe.ru
}

Поступила в Редакцию 16 декабря 2019 г.

В окончательной редакции 20 февраля 2020 г.

Принята к публикации 20 февраля 2020 г.

Разработан метод анализа модификации спонтанного излучения в структурах с цилиндрической симметрией. Выведен метод матриц рассеяния для цилиндрических структур. На основе матриц рассеяния получены правила квантования электромагнитного поля (S-квантование) в цилиндрических системах. Получены общие выражения для темпа излучательной рекомбинации эмиттера, находящегося в произвольной точке структуры. Выведены количественные показатели, дающие оценку усилению и подавлению излучательной рекомбинации, которые могут рассматриваться как модовые факторы Парселла. Получено выражение для суммарного фактора Парселла для излучения в направлении, перпендикулярном оси симметрии среды, а также выражение для интегрального (полного) фактора Парселла для эмиттера на оси симметрии.

Ключевые слова: нанофотоника, спонтанная излучательная рекомбинация, эффект Парселла.

DOI: 10.21883/FTP.2020.07.49506.9336

\section{1. Введение}

Оптические структуры с цилиндрической симметрией являются объектами обширных исследований в современной физике. К числу таких объектов относятся оптические волокна [1,2], структуры с нанопроводами [3-5], дисковые резонаторы и многие другие. Особый интерес вызывают эффекты, связанные с взаимодействием света и полупроводниковых нанообъектов в цилиндрических структурах. Так, полупроводниковые квантовые точки на основе InAs/InGaAs используются в качестве активной среды микродисковых лазеров, работающих на оптических модах шепчущих галерей [6,7]. Системы на основе квантовых проводов, обладающие цилиндрической симметрией, являются перспективными для создания источников одиночных фотонов [8].

В таких системах одним из ключевых эффектов является изменение спонтанной излучательной рекомбинации вследствие пространственной локализации света (эффект Парселла [9]), поэтому необходимы методы для вычисления вероятности спонтанной рекомбинации квантового эмиттера в цилиндрической структуре. Существующие методы на основе формализма функций Грина довольно громоздки и требуют большого объема вычислений $[10,11]$.

Перспективным методом расчета вероятности спонтанной эмиссии является метод $S$-квантования $[12,13]$ на основе матриц рассеяния ( $S$-квантование). В данной работе мы последовательно развиваем метод матриц рассеяния для цилиндрически структурированных систем, разрабатываем метод $S$-квантования для систем цилин- дрической симметрии и получаем выражения для спонтанной излучательной рекомбинации квантового эмиттера и для факторов Парселла в таких системах. Развитие данного формализма необходимо для разработки волоконных лазеров, лазеров на микродисках источников одиночных фотонов на основе квантовых проводов.

\section{2. Теория}

\section{1. Основные уравнения}

В общем случае темп спонтанной излучательной рекомбинации оптического перехода, описываемого матричным элементом дипольного момента $\mathbf{d}_{f i}$, может быть вычислен при помощи золотого правила Ферми:

$$
W=\frac{2 \pi}{\hbar} \frac{1}{4} \sum_{p}\left|\mathbf{E}_{p}(\mathbf{r}) \mathbf{d}_{f i}\right|^{2} \delta\left(\hbar \omega-\hbar \omega_{p}\right),
$$

где $\hbar \omega=E_{i}-E_{f}-$ энергия перехода и $\mathbf{E}_{p}(\mathbf{r})-$ комплексная амплитуда электрического поля фотонной моды, нормированной на энергию фотона:

$$
\frac{1}{16 \pi} \int_{V}\left(\varepsilon(\mathbf{r}) \mathbf{E}_{p} \mathbf{E}_{p}^{*}+\mu(\mathbf{r}) \mathbf{H}_{p} \mathbf{H}_{p}^{*}\right) d^{3} \mathbf{r}=\hbar \omega_{p} .
$$

В выражении (2) $\varepsilon(\mathbf{r})$ и $\mu(\mathbf{r})$ представляют пространственные профили диэлектрической и магнитной проницаемости. В формулах (1), (2) предполагается, что монохроматическое электрическое поле выражается через комплексную амплитуду $\mathbf{E}_{p}(\mathbf{r})$ как $\operatorname{Re}\left\{\mathbf{E}_{p}(\mathbf{r}) \exp \left(-i \omega_{p} t\right)\right\}$ и аналогично для магнитного поля. В выражении (1) 
суммирование ведется по оптическим модам с энергиями фотона $\hbar \omega_{p}$; конкретный вид фотонных мод определяется условиями задачи.

В среде с цилиндрической симметрией, в которой $\varepsilon(\mathbf{r})$ и $\mu(\mathbf{r})$ зависят только от расстояния $\rho$ до оси симметрии, комплексная амплитуда поля моды может быть представлена в виде $\mathbf{E}(\mathbf{r})=\mathbf{E}(\rho) \exp \left(i\left(k_{z} z+m \phi\right)\right)$, где $\phi$ и $z-$ соответствующие координаты в цилиндрической системе координат, $k_{z}-$ продольный волновой вектор и $m$ - целое циклическое число. В этом случае оптические моды могут быть представлены в двух видах: мода, в которой отсутствует магнитное поле, направленное вдоль оси симметрии (ТМ-мода), и мода без электрического поля вдоль оси симметрии (ТЕ-мода). Электромагнитное поле может быть разложено в сумму данных волн в однородном цилиндрическом слое. Однако в общем случае ТМ- и ТЕ-волны смешиваются при прохождении через границу цилиндрических слоев [13]. В этой работе мы рассматриваем наиболее важные случаи излучения перпендикулярно оси симметрии (с продольным волновым числом, равным нулю $k_{z}=0$ ), a также излучение в цилиндрические моды с циклическим числом, равным нулю $m=0$. В этих случаях ТМ- и ТЕ-моды, распространяющиеся в цилиндрически симметричной структуре, не смешиваются. Тогда компоненты ТМ-моды в однородном цилиндрическом слое могут быть представлены в виде:

$$
\begin{gathered}
E_{z}^{\mathrm{TM}}=A_{\mathrm{TM}}^{+,-} \frac{k_{\rho}}{K} H_{m}^{(1,2)}\left(k_{\rho} \rho\right), \\
H_{\phi}^{\mathrm{TM}}=A_{\mathrm{TM}}^{+,-} i \sqrt{\frac{\varepsilon}{\mu}} H_{m}^{\prime(1,2)}\left(k_{\rho} \rho\right), \\
H_{\rho}^{\mathrm{TM}}=A_{\mathrm{TM}}^{+,-} \sqrt{\frac{\varepsilon}{\mu}} \frac{k_{\phi m}}{k_{\rho}} H_{m}^{(1,2)}\left(k_{\rho} \rho\right), \\
E_{\rho}^{\mathrm{TM}}=A_{\mathrm{TM}}^{+,-} i \frac{k_{z}}{K} H_{m}^{(1,2)}\left(k_{\rho} \rho\right) .
\end{gathered}
$$

Здесь введены обозначения для волновых чисел: $K=\sqrt{\varepsilon \mu} \omega_{p} / c, k_{\phi m}=m / \rho$ и $k_{\rho}=\sqrt{K^{2}-k_{z}^{2}}$; для мод c $m \neq 0$ в выражениях (3) нужно положить $k_{z}=0$ и $k_{\rho}=K$. Функции Ганкеля первого рода $H_{m}^{(1)}$ дают расходящиеся от оси симметрии цилиндрические волны (на что указывает индекс + у коэффициентов $A$ ), а функции Ганкеля второго рода $H_{m}^{(2)}$ соответствуют сходящимся цилиндрическим волнам. Компоненты ТЕ-мод могут быть представлены в виде:

$$
\begin{gathered}
E_{\phi}^{\mathrm{TE}}=-A_{\mathrm{TE}}^{+,-} i H_{m}^{(1,2)}\left(k_{\rho} \rho\right), \\
E_{\rho}^{\mathrm{TE}}=-A_{\mathrm{TE}}^{+,-}-\frac{k_{\phi m}}{k_{\rho}} H_{m}^{(1,2)}\left(k_{\rho} \rho\right), \\
H_{z}^{\mathrm{TE}}=A_{\mathrm{TE}}^{+,-} \sqrt{\frac{\varepsilon}{\mu}} \frac{k_{\rho}}{K} H_{m}^{(1,2)}\left(k_{\rho} \rho\right), \\
H_{\rho}^{\mathrm{TE}}=A_{\mathrm{TE}}^{+,-} i \sqrt{\frac{\varepsilon}{\mu}} \frac{k_{z}}{K} H_{m}^{(1,2)}\left(k_{\rho} \rho\right) .
\end{gathered}
$$

Из непрерывности тангенциальных компонент электрического и магнитного полей на цилиндрической гра- нице слоев можно получить коэффициенты отражения и прохождения, которые мы определяем через отношения электрических компонент, $E_{z}^{\mathrm{TM}}$ для ТМ-мод и $E_{\phi}^{\mathrm{TE}}$ для ТЕ-мод (см. Приложение I).

Коэффициенты прохождения и отражения для данного типа мод могут быть собраны в матрицу размерности 2, которая связывает волны, падающие на структуру, с волнами, исходящими от структуры:

$$
\begin{gathered}
\left(\frac{E_{\text {out }}^{-}}{E_{\text {out }}^{+}}\right)=\hat{S}_{\text {str }}\left(\frac{E_{\text {in }}^{-}}{E_{\text {in }}^{+}}\right), \\
\hat{S}_{\text {str }}=\left(\begin{array}{ll}
t^{-} & r^{+} \\
r^{-} & t^{+}
\end{array}\right) .
\end{gathered}
$$

В выражении (5а) знаком плюс (минус) обозначены амплитуды расходящихся (сходящихся) цилиндрических волн; коэффициенты в (5б) обозначаются знаками плюс или минус исходя из характера падающей волны. Матрицы такого вида называются матрицами рассеяния $(S$-матрицами); в данной работе мы будем развивать формализм матриц рассеяния для расчета профиля электромагнитного поля, спонтанной рекомбинации и фактора Парселла в цилиндрических структурах.

Матрицы рассеяния для структуры с чередующимися цилиндрическими границами и однородными цилиндрическими слоями могут быть получены последовательным применением следующей процедуры:

$$
\begin{aligned}
& t^{-}=\frac{t_{2}^{-} t_{1}^{-}}{1-r_{1}^{+} r_{2}^{-}}, \quad t^{+}=t_{1}^{+} t_{2}^{+}+\frac{t_{1}^{+} r_{2}^{-} r_{1}^{+} t_{2}^{+}}{1-r_{1}^{+} r_{2}^{-}}, \\
& r^{+}=r_{2}^{+}+\frac{t_{2}^{-} r_{1}^{+} t_{2}^{+}}{1-r_{1}^{+} r_{2}^{-}}, r^{-}=r_{1}^{-}+\frac{t_{1}^{+} r_{2}^{-} t_{1}^{-}}{1-r_{1}^{+} r_{2}^{-}} .
\end{aligned}
$$

Здесь индекс 1 обозначает коэффициенты, составляющие матрицу рассеяния для внешней части цилиндрической структуры, и индекс 2 обозначает внутреннюю часть. Формулы (6) могут быть получены наглядно через суммирование возможных путей прохождения фотона сквозь составную структуру или отражения фотона от структуры. Предположим, что в центре рассматриваемой цилиндрической структуры находится однородный цилиндр. Так как функции Ганкеля в формулах (3), (4) выражаются через функции Бесселя $H_{m}^{(1,2)}(x)=J_{m}(x) \pm i Y_{m}(x)$ и функции $Y_{m}(x)$ расходятся в нуле, условие конечности поля на оси симметрии структуры приводит к равенству коэффициентов при сходящейся и расходящейся волнах в центральном цилиндре. Отсюда следуют выражения для коэффициентов отражения от оси симметрии:

$$
\begin{aligned}
& r_{\alpha x \mathrm{TM}}\left(k_{\rho \mathrm{int}} \rho\right)=\frac{H_{m}^{(1)}\left(k_{\rho \mathrm{int}} \rho\right)}{H_{m}^{(2)}\left(k_{\rho \mathrm{int}} \rho\right)}, \\
& r_{\alpha x \mathrm{TE}}\left(k_{\rho \mathrm{int}} \rho\right)=\frac{H_{m}^{\prime(1)}\left(k_{\rho \mathrm{int}} \rho\right)}{H_{m}^{\prime(2)}\left(k_{\rho \mathrm{int}} \rho\right)},
\end{aligned}
$$

где волновой вектор $k_{\rho \mathrm{int}}=\sqrt{\varepsilon_{\mathrm{int}} \mu_{\mathrm{int}} \omega_{p}^{2} / c^{2}-k_{z}^{2}}$ определяется при помощи оптических параметров $\varepsilon_{\text {int }}, \mu_{\text {int }}$, центрального цилиндра. 
Особый интерес представляет нахождение полного коэффициента отражения от структуры с цилиндрической симметрией. Если $r_{\mathrm{stTM} / \mathrm{TE}}^{ \pm}$и $t_{\mathrm{stTM} / \mathrm{TE}}^{ \pm}-$коэффициенты отражения и прохождения для составной структуры, обрамляющей центральный цилиндр, то полный коэффициент отражения дается формулой

$$
r_{\mathrm{TM} / \mathrm{TE}}=r_{\mathrm{stTM} / \mathrm{TE}}^{-}+\frac{t_{\mathrm{stTM} / \mathrm{TE}}^{+} r_{\alpha x \mathrm{TM} / \mathrm{TE}} t_{\mathrm{stTM} / \mathrm{TE}}^{-}}{1-r_{\mathrm{stTM} / \mathrm{TE}}^{+} r_{\alpha x \mathrm{TM} / \mathrm{TE}}} .
$$

Для расчета темпа спонтанной рекомбинации по формуле (1) необходимо определить процедуру квантования для квазидискретных фотонных мод. Поместим цилиндрическую структуру в цилиндр квантования радиуса $R$ и высоты $L_{z}$ по аналогии с обычно используемым параллелепипедом квантования. Аналогично методу $S$-квантования, разработанному для плоских слоистых структур $[8,9]$, будем считать условием квантования фотонных мод равенство единице собственных чисел полной матрицы рассеяния цилиндра квантования, содержащего рассматриваемую структуру:

$$
\beta_{\mathrm{TM} / \mathrm{TE}}=1 .
$$

С физической точки зрения уравнение (9) требует равенства электрического поля в волнах, падающей на структуру и исходящей от структуры на границе области квантования. Для случая несмешивающихся ТМ- и ТЕ-мод матрица рассеяния структуры - это коэффициент отражения от структуры, определяемый формулой (8). Тогда полная матрица рассеяния цилиндра квантования, содержащего цилиндрическую структуру, может быть выражена через коэффициенты отражения от структуры (8) и от оси симметрии (7), так что уравнение (9) выражается следующим образом:

$$
\frac{r_{\alpha x \mathrm{TM} / \mathrm{TE}}\left(k_{\rho \mathrm{ext}} R\right)}{r_{\alpha x \mathrm{TM} / \mathrm{TE}}\left(k_{\rho \mathrm{ext}} \rho_{0}\right)} r_{\mathrm{TM} / \mathrm{TE}}=1
$$

Здесь волновое число $k_{\rho \mathrm{ext}}=\sqrt{\varepsilon_{\mathrm{ext}} \mu_{\mathrm{ext}} \omega_{p}^{2} / c^{2}-k_{z}^{2}}$ выражается через оптические параметры внешней среды и $\rho_{0}$ - внешний радиус структуры. Используя асимптотику функций Ганкеля при большом аргументе, условие квантования (9), (10) в пределе большого радиуса цилиндра квантования $R \rightarrow \infty$ может быть записано в виде

$$
\exp \left(i\left(2 k_{\rho \mathrm{ext}} R-m \pi-\frac{\pi}{2}\right)\right) \frac{r_{\mathrm{TM} / \mathrm{TE}}}{r_{\alpha x \mathrm{TM} / \alpha x \mathrm{TE}}\left(k_{\rho \mathrm{ext}} \rho_{0}\right)}=1 .
$$

Это уравнение на собственные фотонные состояния цилиндра квантования может иметь решения при вещественных частотах $\hbar \omega_{p}$, если цилиндрическая структура не содержит поглощающих элементов и, соответственно, модуль коэффициента отражения от структуры равен единице $\left|r_{\mathrm{TM} / \mathrm{TE}}\right|=1$. Тогда уравнение (11) сводится к равенству фазы комплексного числа слева следующей величине $2 \pi N_{\rho}$, где $N_{\rho}$ - целое число. Тогда дифференциал числа фотонных состояний $d N_{\rho}$, связанный с радиальным распределением поля, может быть приведен к следующему виду:

$$
d N_{\rho}=\left(\frac{R}{\pi}+\left.\frac{1}{2 \pi} \frac{\partial \alpha_{\mathrm{TM} / \mathrm{TE}}}{\partial k_{\rho \mathrm{ext}}}\right|_{k z=\mathrm{const}}\right) d k_{\rho \mathrm{ext}} \underset{R \rightarrow \infty}{\longrightarrow} \frac{R}{\pi} d k_{\rho \mathrm{ext}},
$$

где $\alpha_{\mathrm{TM} / \mathrm{TE}}-$ фаза отношения коэффициентов отражения в формуле (11). Выражение (12) показывает, что в приделе большого радиуса цилиндра квантования процедура $S$-квантования дает ту же плотность фотонных мод, что и в случае однородной трубы большого диаметра с идеально проводящими стенками.

Так как рассматриваемая структура однородна в направлении оси симметрии, дифференциал числа фотонных мод, связанный с продольным распределением поля $d N_{z}$, может быть получен через стандартные циклические условия на торцах цилиндра квантования: $d N_{z}=\left(I_{z} /(2 \pi)\right) d k_{z}$. Тогда полный дифференциал числа фотонных мод с фиксированным циклическим числом $m$, описываемых продольным волновым числом $k_{z}$ и поперечным волновым числом $k_{\rho \text { ext }}$ во внешней среде, может быть записан как

$$
\begin{aligned}
d N & =d N_{\rho} d N_{z}=\frac{R}{\pi} \frac{L_{z}}{2 \pi} d k_{\rho \mathrm{ext}} d k_{z} \\
& =\varepsilon_{\mathrm{ext}} \mu_{\mathrm{ext}} \frac{\omega_{p}}{c^{2} \hbar} d \hbar \omega_{p} d \theta_{\mathrm{ext}},
\end{aligned}
$$

где угол $\theta_{\text {ext }}$ задается соотношением $k_{\rho \text { ext }}=K_{\text {ext }} \sin \left(\theta_{\text {ext }}\right)$, $k_{z}=K_{\text {ext }} \cos \left(\theta_{\text {ext }}\right)$. Можно показать, что на больших расстояниях цилиндрические волны (3), (4) представляют плоские волны, распространяющиеся под углом $\theta_{\text {ext }}$ к оси симметрии. При выводе формулы (13) мы использовали соотношение $d k_{\rho \text { ext }} d k_{z}=K_{\text {ext }} d \theta_{\text {ext }} d K_{\text {ext }}$.

Для расчета спонтанной рекомбинации необходимо нормировать фотонные состояния. Поле за пределами структуры будет состоять из суммы падающих и отраженных бегущих волн (уравнения (3), (4)), связанных коэффициентами отражения (8). Электрическое поле за пределами структуры представлено в явном виде в Приложсении II, формулы (П.II.1), (П.IІ.2). Для данных цилиндрических мод интеграл в выражении (2) расходится при стремлении радиуса цилиндра квантования к бесконечности, поэтому для нормировки можно использовать асимптотическую форму цилиндрических волн для больших расстояний от оси симметрии. Тогда нормировка моды на энергию фотона дает модуль амплитуды бегущих волн

$$
\left|A_{\mathrm{TM} / \mathrm{TE}}\right|^{2}=k_{\rho \mathrm{ext}} \frac{\pi \hbar \omega_{p}}{\varepsilon_{\mathrm{ext}} L_{z} R}
$$

\section{2. Темп спонтанной рекомбинации эмиттера в цилиндрической структуре}

Теперь мы можем найти выражение для темпа спонтанной излучательной рекомбинации в цилиндрические фотонные моды с определенным циклическим числом $m$. 
В выражении (1) для золотого правила Ферми при помощи формулы (13) заменим суммирование по фотонным модам на интегрирование

$$
\begin{aligned}
d w_{\mathrm{TM} / \mathrm{TE}}^{m} & =W_{\mathrm{TM} / \mathrm{TE}}^{m} d N \\
& =\frac{1}{\hbar} \frac{R L_{z}}{4 \pi} \varepsilon_{\mathrm{ext}} \mu_{\mathrm{ext}} \frac{\omega}{c^{2} \hbar}\left|\mathbf{E}_{\mathrm{TM} / \mathrm{TE}}^{m}\left(\rho, \omega_{p}=\omega\right) \mathbf{d}_{f i}\right|^{2} d \theta_{\mathrm{ext}}
\end{aligned}
$$

Теперь в (15) выразим нормированное электрическое поле $\mathbf{E}_{\mathrm{TM} / \mathrm{TE}}^{m}$ через амплитуду бегущих волн во внешней среде, формула (14), и безразмерный профиль поля $\tilde{\mathbf{E}}_{\mathrm{TM} / \mathrm{TE}}^{m}$ :

$$
\begin{aligned}
& d w_{\mathrm{TM} / \mathrm{TE}}^{m}=\mu_{\mathrm{ext}} \sqrt{\varepsilon_{\mathrm{ext}} \mu_{\mathrm{ext}}} \\
& \times \frac{\omega^{3}}{4 c^{3} \hbar}\left|\tilde{\mathbf{E}}_{\mathrm{TM} / \mathrm{TE}}^{m}\left(\rho, \omega_{p}=\omega\right) \mathbf{d}_{f i}\right|^{2} \sin \theta_{\mathrm{ext}} d \theta_{\mathrm{ext}} .
\end{aligned}
$$

Безразмерный профиль $\tilde{\mathbf{E}}_{\mathrm{TM} / \mathrm{TE}}^{m}$ рассчитывается исходя из условия падения на структуру цилиндрической волны с единичной амплитудой, т. е. в формулах (П.ІІ.1), (П.ІІ.2) нужно подставить $A_{\mathrm{TM} / \mathrm{TE}}=1$. Можно видеть, что геометрические параметры цилиндра квантования $R$ и $L_{z}$ сократились в выражении (16), как и следовало ожидать.

Для мод с циклическим числом $m \neq 0$ формула (16) может быть использована только для расчета спонтанной рекомбинации в направлении, поперечном оси симметрии $k_{z}=0, \theta_{\text {ext }}=\pi / 2$. Для ТЕ- и ТМ-мод с $m=0$, обычно имеющих максимальное поле на оси симметрии, формула (16) может быть использована для расчета полного темпа рекомбинации $w_{\mathrm{TM} / \mathrm{TE}}^{m=0}$ в эти моды через интегрирование по углу $\theta_{\text {ext }}$ от 0 до $\pi$.

Полный темп спонтанной излучательной рекомбинации во внешний угол $d \theta_{\text {ext }}$ для поперечного излучения $\left(\theta_{\text {ext }}=\pi / 2\right)$ дается суммированием выражения (16) по поляризации и по циклическим числам $m$ :

$$
\begin{aligned}
& d w=\sum_{\substack{J=\mathrm{TM}, \mathrm{TE} \\
m=-\infty \ldots+\infty}} d w_{J}^{m} \\
& =\mu_{\mathrm{ext}} \sqrt{\varepsilon_{\mathrm{ext}} \mu_{\mathrm{ext}}} \frac{\omega^{3}}{4 c^{3} \hbar}\left\{\sum_{\substack{J=\mathrm{TM}, \mathrm{TE} \\
m=-\infty \ldots+\infty}}\left|\tilde{\mathbf{E}}_{J}^{m} \mathbf{d}_{f i}\right|^{2}\right\} \sin \theta_{\mathrm{ext}} d \theta_{\mathrm{ext}} \\
& =\mu_{\mathrm{ext}} \sqrt{\varepsilon_{\mathrm{ext}} \mu_{\mathrm{ext}}} \frac{\omega^{3}}{4 c^{3} \hbar} \\
& \times\left\{\sum_{J=\mathrm{TM}, \mathrm{TE}}\left|\tilde{\mathbf{E}}_{J}^{m=0} \mathbf{d}_{f i}\right|^{2}+2 \sum_{\substack{J=\mathrm{TM}, \mathrm{TE} \\
m=1 \ldots+\infty}}\left|\tilde{\mathbf{E}}_{J}^{m} \mathbf{d}_{f i}\right|^{2}\right\} \sin \theta_{\mathrm{ext}} d \theta_{\mathrm{ext}} .
\end{aligned}
$$

Если матрица рассеяния $\hat{S}_{\mathrm{str}}(5)$ для структуры, обрамляющей центральный цилиндр, рассчитана по вышеописанной процедуре, то безразмерный профиль электрического поля $\tilde{\mathbf{E}}_{\mathrm{TM} / \mathrm{TE}}^{m}$ внутри цилиндра может быть представлен в виде

$$
\begin{aligned}
& \tilde{E}_{z}^{\mathrm{TM} \text { int }}=2 \frac{k_{\rho \mathrm{ext}}}{K_{\mathrm{ext}}} \frac{H_{m}^{(2)}\left(k_{\rho \mathrm{ext}} \rho_{0}\right)}{H_{m}^{(2)}\left(k_{\rho \mathrm{int}} \rho_{\mathrm{int}}\right)} \\
& \times \frac{t_{\text {stTM }}^{-}}{1-r_{\text {stTM }}^{+} r_{\alpha x \mathrm{TM}}\left(k_{\rho \text { int }} \rho_{\text {int }}\right)} J_{m}\left(k_{\rho \text { int }} \rho\right), \\
& \tilde{E}_{\rho}^{\mathrm{TM} \text { int }}=i 2 \frac{k_{\rho \mathrm{ext}}}{k_{\rho \mathrm{int}}} \frac{k_{z}}{K_{\mathrm{ext}}} \frac{H_{m}^{(2)}\left(k_{\rho \mathrm{ext}} \rho_{0}\right)}{H_{m}^{(2)}\left(k_{\rho \mathrm{int}} \rho_{\mathrm{int}}\right)} \\
& \times \frac{t_{\mathrm{stTM}}^{-}}{1-r_{\mathrm{stTM}}^{+} r_{\alpha x \mathrm{TM}}\left(k_{\rho \mathrm{int}} \rho_{\mathrm{int}}\right)} J_{m}^{\prime}\left(k_{\rho \mathrm{int}} \rho\right), \\
& \tilde{E}_{\phi}^{\mathrm{TE} \text { int }}=-i 2 \frac{H_{m}^{\prime(2)}\left(k_{\rho \mathrm{ext}} \rho_{0}\right)}{H_{m}^{\prime(2)}\left(k_{\rho \text { int }} \rho_{\text {int }}\right)} \\
& \times \frac{t_{\mathrm{stTM}}^{-}}{1-r_{\mathrm{stTE}}^{+} r_{\alpha x \mathrm{TE}}\left(k_{\rho \mathrm{int}} \rho_{\mathrm{int}}\right)} J_{m}^{\prime}\left(k_{\rho \mathrm{int}} \rho\right), \\
& \tilde{E}_{\rho}^{\mathrm{TE} \mathrm{int}}=-2 m \frac{H_{m}^{\prime(2)}\left(k_{\rho \mathrm{ext}} \rho_{0}\right)}{H_{m}^{\prime(2)}\left(k_{\rho \mathrm{int}} \rho_{\mathrm{int}}\right)} \\
& \times \frac{t_{\mathrm{stTM}}^{-}}{1-r_{\mathrm{stTE}}^{+} r_{\alpha x \mathrm{TE}}\left(k_{\rho \mathrm{int}} \rho_{\mathrm{int}}\right)} \frac{J_{m}\left(k_{\rho \mathrm{int}} \rho\right)}{k_{\rho \mathrm{int}} \rho},
\end{aligned}
$$

где $\rho_{\text {int }}-$ радиус центрального цилиндра, $\rho_{0}$ - внешний радиус структуры; $k_{\rho \text { int }}$ и $k_{\rho \text { ext }}-$ поперечные волновые числа внутри центрального цилиндра и во внешней среде соответственно.

Подставляя (18) в формулу (16), можно увидеть, что темп спонтанной рекомбинации будет усиливаться при уменьшении фактора в знаменателе в (18). С качественной точки зрения данное условие означает положительную интерференцию цилиндрических волн, бегущих по замкнутому пути внутри центрального цилиндра. Подставляя выражения коэффициентов отражения и прохождения для цилиндрической границы двух диэлектриков, приведенные в Приложении I, в выражения (18), можно получить аналитические формулы для темпа спонтанной рекомбинации эмиттера внутри однородного цилиндра.

\section{3. Темп спонтанной рекомбинации эмиттера на оси симметрии}

Важнейшим частным случаем является излучательная спонтанная рекомбинация дипольного перехода, находящегося на оси симметрии структуры и сонаправленного оси симметрии. Рассматривая асимптотику электромагнитных полей при $\rho \rightarrow 0$, можно показать, что только ТМ-мода с $m=0$ имеет ненулевое поле вдоль оси симметрии $E_{z}^{\mathrm{TM}, m=0}(\rho=0) \neq 0$ и, следовательно, только такая мода будет взаимодействовать с подобным квантовым переходом. Безразмерное электрическое поле (18) 
на оси симметрии имеет вид

$$
\begin{aligned}
\tilde{\mathbf{E}}_{\mathrm{TM}}^{m=0}(\rho=0)= & 2 \frac{k_{\rho \mathrm{ext}}}{K_{\mathrm{ext}}} \frac{H_{m=0}^{(2)}\left(k_{\rho \mathrm{ext}} \rho_{0}\right)}{H_{m=0}^{(2)}\left(k_{\rho \mathrm{int}} \rho_{\mathrm{int}}\right)} \\
& \times \frac{t_{\mathrm{stTM}}^{-}}{1-r_{\mathrm{stTM}}^{+} r_{\alpha x \mathrm{TM}}\left(k_{\rho \mathrm{int}} \rho_{\mathrm{int}}\right)} \mathbf{e}_{z},
\end{aligned}
$$

где $\mathbf{e}_{z}-$ единичный вектор вдоль оси симметрии. Тогда для квантового перехода на оси симметрии, описываемого матричным элементом дипольного момента $\mathbf{d}_{f i}=\left(\mathbf{d}_{f i}\right)_{z} \mathbf{e}_{z}$, подстановка (19) в (17) дает

$$
\begin{aligned}
d w= & \mu_{\mathrm{ext}} \sqrt{\varepsilon_{\mathrm{ext}} \mu_{\mathrm{ext}}} \frac{\omega^{3}\left|\left(\mathbf{d}_{f i}\right)_{z}\right|^{2}}{c^{3} \hbar} \mid \frac{H_{m=0}^{(2)}\left(k_{\rho \mathrm{ext}} \rho_{0}\right)}{H_{m=0}^{(2)}\left(k_{\rho \mathrm{int}} \rho_{\mathrm{int}}\right)} \\
& \times\left.\frac{t_{\mathrm{stTM}}^{-}}{1-r_{\mathrm{stTM}}^{+} r_{\alpha x \mathrm{TM}}\left(k_{\rho \mathrm{int}} \rho_{\mathrm{int}}\right)}\right|^{2} \sin ^{3}\left(\theta_{\mathrm{ext}}\right) d \theta_{\mathrm{ext}} .
\end{aligned}
$$

Интегрирование этого выражения по углу $\theta_{\text {ext }}$ от 0 до $\pi$ дает полный темп излучательной рекомбинации в волны во внешней среде для дипольного перехода, находящегося на оси симметрии и сонаправленного ей.

Для проверки правильности выкладок заменим цилиндрическую структуру на однородный цилиндр. Тогда в выражении (20): $k_{\rho \text { ext }}=k_{\rho \text { int }}, \rho_{0}=\rho_{\text {int }}, r_{\text {stTM }}^{+}=0$ и $t_{\mathrm{stTM}}^{-}=1$. Интегрируя по углу и учитывая, что $\int_{0}^{\pi} \sin ^{3} \theta d \theta=4 / 3$, получаем

$$
w=\frac{4}{3} \mu \sqrt{\varepsilon \mu} \frac{\omega^{3}}{\hbar c^{3}}\left|\left(\mathbf{d}_{f i}\right)_{z}\right|^{2}
$$

Это выражение совпадает с классическим выражением для темпа спонтанной излучательной рекомбинации дипольного перехода в однородной среде. Таким образом, мы показали, что в тривиальном случае дипольного перехода в однородной среде метод $S$-квантования в цилиндрической геометрии дает тот же результат, что и привычный подход с использованием параллелепипеда квантования и циклических граничных условий.

\section{4. Модовый фактор Парселла}

Дадим количественную характеристику тому, каким образом цилиндрическая структура модифицирует темп спонтанной излучательной рекомбинации. Рассмотрим эмиттер внутри центрального цилиндра составной цилиндрической структуры и введем соотношение $F_{\mathrm{MT} / \mathrm{TE}}^{m}=d w_{\mathrm{TM} / \mathrm{TE}}^{m} / d \tilde{w}_{\mathrm{TM} / \mathrm{TE}}^{m}$, где числитель дается сочетанием формул (16) и (18), а $d \tilde{w}_{\mathrm{TM} / \mathrm{TE}}^{m}-$ часть темпа спонтанной рекомбинации эмиттера в однородной среде для излучения посредством цилиндрических волн внутри дифференциального угла $d \theta_{\text {int. }}$. Величину $d \tilde{w}_{\mathrm{TM} / \mathrm{TE}}^{m}$ можно получить подстановкой безразмерного профиля поля в однородной среде, формулы (П.ІІ.3), (П.ІІ.4) в Приложении II, в выражение (16). Учитывая, что дифференциальный угол излучения внутри центрального цилиндра $d \theta_{\text {int }}$ связан с внешним углом $d \theta_{\text {ext }}$ законом Снеллиуса $K_{\text {int }} \sin \left(\theta_{\text {int }}\right) d \theta_{\text {int }}=K_{\text {ext }} \sin \left(\theta_{\text {ext }}\right) d \theta_{\text {ext }}$, получим

$$
\begin{gathered}
F_{\mathrm{TM}}^{m}=\frac{\varepsilon_{\text {int }} k_{\rho \text { ext }}^{2}}{\varepsilon_{\text {ext }}^{2} k_{\rho \text { int }}^{2}}\left|\frac{H_{m}^{(2)}\left(k_{\rho \text { ext }} \rho_{0}\right)}{H_{m}^{(2)}\left(k_{\rho \text { int }} \rho_{\text {int }}\right)}\right|^{2}\left|\frac{t_{\text {stTM }}^{-}}{1-r_{\text {stTM }}^{+} r_{\alpha x \mathrm{TM}}\left(k_{\rho \text { int }} \rho_{\text {int }}\right)}\right|^{2}, \\
F_{\mathrm{TE}}^{m}=\frac{\mu_{\text {ext }}}{\mu_{\text {int }}}\left|\frac{H_{m}^{(2)}\left(k_{\rho \text { ext }} \rho_{0}\right)}{H_{m}^{\prime(2)}\left(k_{\rho \text { int }} \rho_{\text {int }}\right)}\right|^{2}\left|\frac{t_{\text {stTE }}^{-}}{1-r_{\text {stTE }}^{+} r_{\alpha x \mathrm{TE}}\left(k_{\rho \text { int }} \rho_{\text {int }}\right)}\right|^{2} .
\end{gathered}
$$

Величины $F_{\mathrm{TM} / \mathrm{TE}}^{m}$ показывают, насколько усиливается или подавляется способность эмиттера излучать свет под определенным углом во внешнюю среду, и, соответственно, эти величины можно рассматривать как модовые факторы Парселла. Для мод с циклическим числом $m \neq 0$ формулы (22) должны использоваться для оценки модификации спонтанного излучения в направлении, поперечном оси симметрии. Для ТМ- и ТЕ-мод с $m=0$ формулы (22) дают модовые факторы Парселла для любого угла распространения во внешней среде. Можно заметить, что факторы (22) не зависят от положения эмиттера и направления дипольного перехода $\mathbf{d}_{f i}$, тогда как в формулах для темпа рекомбинации (16) данные зависимости присутствуют. Как можно видеть из формул (3), (4), при излучении в поперечном направлении $\left(k_{z}=0\right)$ факторы $F_{\mathrm{TM}}^{m}$ для ТМ-мод описывают модификацию спонтанного излучения диполей, направленных вдоль оси симметрии, тогда как факторы $F_{\mathrm{TM}}^{m}$ определяют излучение диполей, лежащих в плоскости, перпендикулярной оси симметрии.

\section{5. Полный фактор Парселла}

Для расчета полного фактора Парселла с учетом любого направления распространения излучения и произвольного положения эмиттера необходимо разработать формализм, учитывающий смешивание ТМ- и ТЕ-мод, что будет темой следующей работы.

Подход, представленный в данной работе, достаточен для расчета полного фактора Парселла для излучения в поперечном направлении. Данная величина дается отношением полного темпа спонтанного излучения в поперечном направлении из структуры $d w$, вычисляемого с помощью формулы (17), и соответствующей величины для однородного пространства $d \tilde{w}$ :

$$
\begin{aligned}
& F_{\theta=\pi / 2}^{\mathrm{tot}}= \frac{d w}{d \tilde{w}} \\
&=\frac{\mu_{\mathrm{ext}}}{\mu_{\mathrm{int}}} \frac{\sum_{J=\mathrm{TM}, \mathrm{TE}}\left|\tilde{\mathbf{E}}_{J}^{m=0} \mathbf{d}_{f i}\right|^{2}+2 \sum_{\substack{J=\mathrm{TM}, \mathrm{TE} \\
m=1 \ldots+\infty}}\left|\tilde{\mathbf{E}}_{J 0}^{m=0} \mathbf{d}_{f i} \mathbf{d}_{f i}\right|^{2}+2 \sum_{\substack{J=\mathrm{TM}, \mathrm{TE} \\
m=1 \ldots+\infty}}\left|\tilde{\mathbf{E}}_{J 0}^{m} \mathbf{d}_{f i}\right|^{2}}{\sum^{2}},
\end{aligned}
$$

где безразмерные профили $\tilde{\mathbf{E}}_{J 0}^{m}$ представлены в формулах (П.IІ.3), (П.ІІ.4). Здесь учитывалось что, согласно 

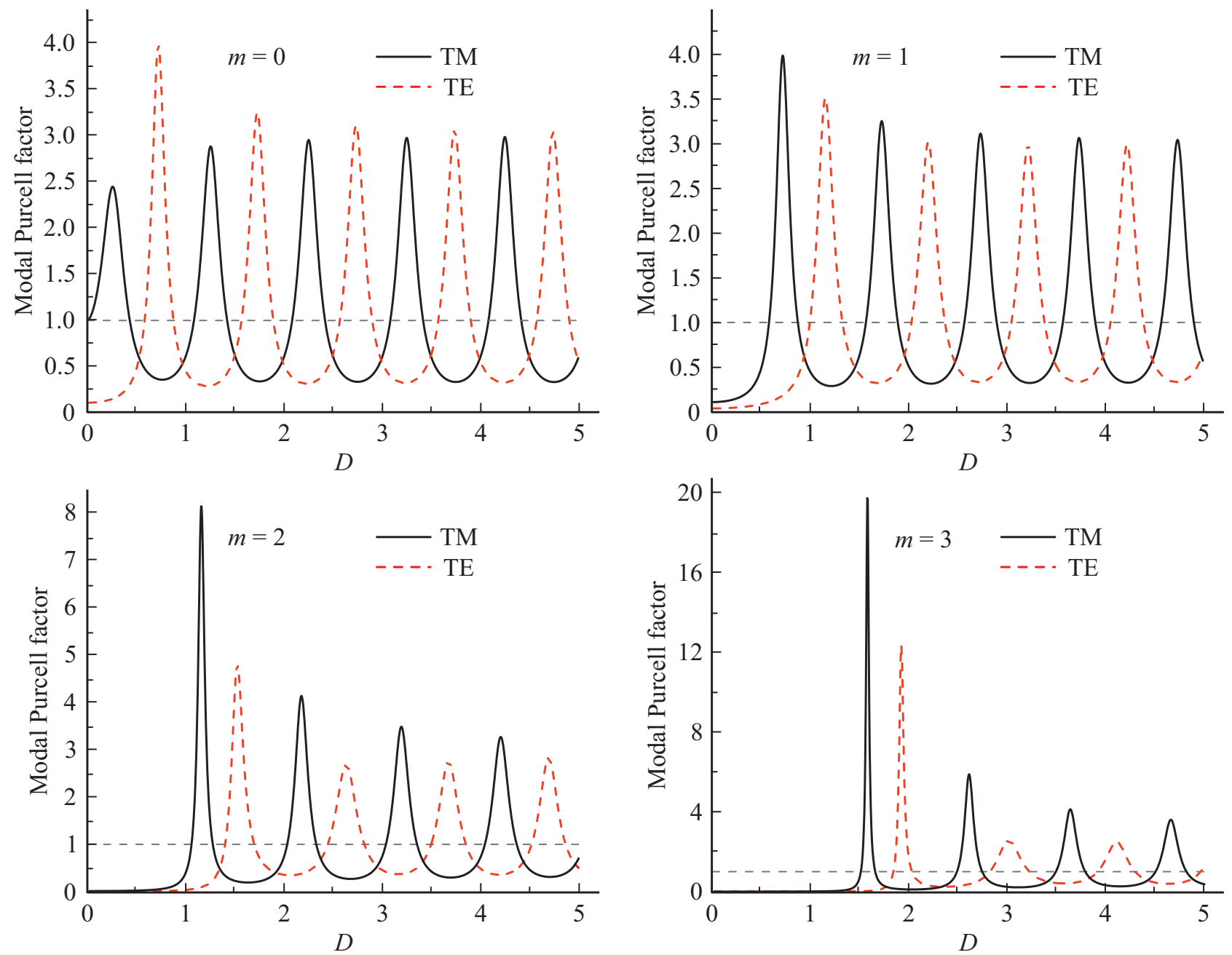

Рис. 1. Модовый фактор Перселла (22) для поперечного излучения $\left(\theta_{\mathrm{ext}}=\pi / 2, k_{z}=0\right)$ для диэлектрического цилиндра с параметрами $\varepsilon_{\text {int }}=9, \mu_{\text {int }}=1$ как функция приведенного диаметра $D=2 \rho_{0} / \lambda_{\text {int }}$, где $\lambda_{\text {int }}=2 \pi c /\left(\sqrt{\varepsilon_{\text {int }}} \omega\right)$ - длина волны в материале цилиндра. В качестве внешней среды взят воздух $\left(\varepsilon_{\text {int }}=1\right)$.
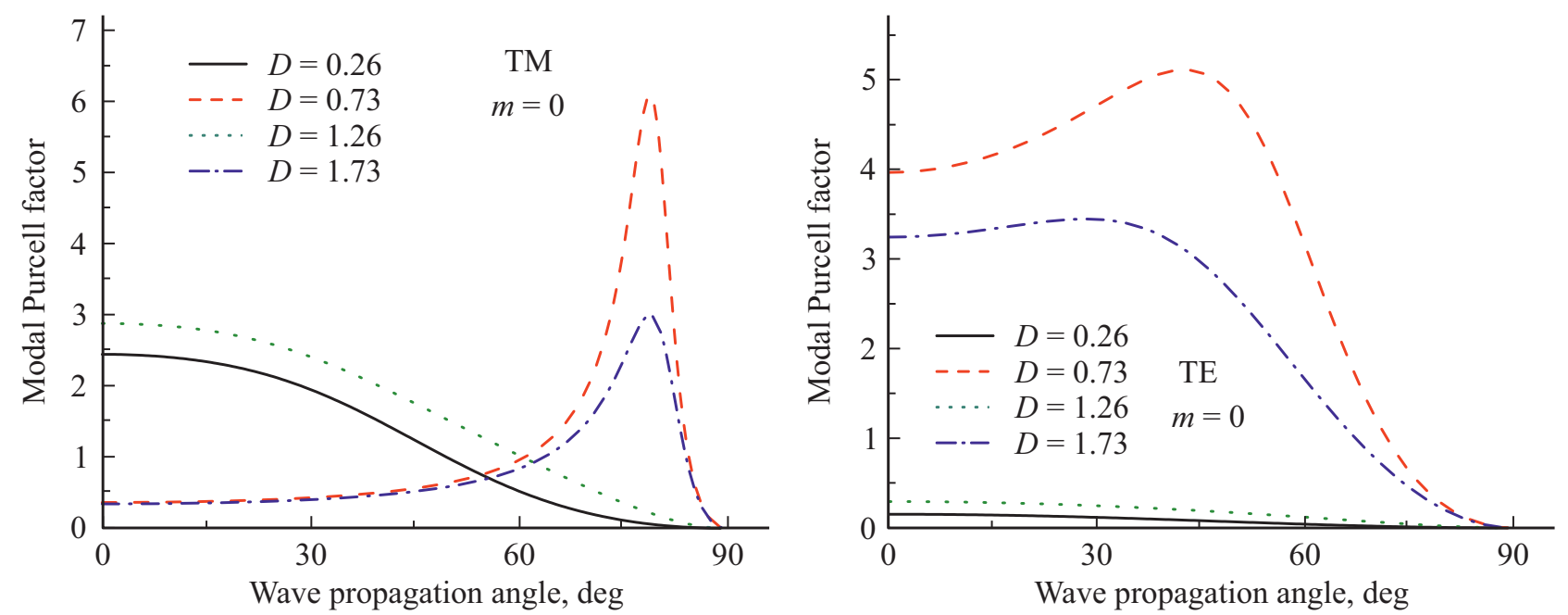

Рис. 2. Модовый фактор Перселла (22) диэлектрического цилиндра для циклического числа $m=0$ как функция угла распространения волн во внешней среде; угол отсчитывается от направления, поперечного оси цилиндра. Рассмотрены приведенные диаметры цилиндра $D=0.26,0.73,1.26,1.23$, соответствующие пикам на рис. 1 . 
закону Снеллиуса, внутренний и внешний дифференциальные углы излучения при излучении в поперечном направлении связаны как $\sqrt{\varepsilon_{\text {int }} \mu_{\text {int }}} d \theta_{\text {int }}=\sqrt{\varepsilon_{\text {ext }} \mu_{\text {ext }}} d \theta_{\text {ext }}$. Для расчета полного фактора Парселла для дипольного перехода, находящегося в центральном цилиндре, в (23) необходимо подставить выражения для безразмерных полей из (18).

Можно видеть, что в общем случае полный фактор Парселла будет зависеть от положения и поляризации дипольного перехода, в отличие от модовых факторов (22). Важным частным случаем является нахождение эмиттера на оси симметрии структуры. Согласно асимптотике цилиндрических полей, дипольный переход на оси симметрии будет взаимодействовать только с модами $m=0$ и $m= \pm 1$. Так, если диполь сонаправлен оси симметрии $\mathbf{d}_{f i}\left(\mathbf{d}_{f i}\right)_{z} \mathbf{e}_{z}$, то полный фактор Парселла для любого угла совпадает с модовым фактором Парселла ТМ-моды с $m=0$. Полный интегральный фактор Парселла определим как отношение полного темпа излучательной рекомбинации в распространяющиеся во внешней среде моды к темпу рекомбинации эмиттера в однородной внешней среде (формула (21)). В случае дипольного перехода на оси симметрии и сонаправленного оси полный темп излучательной рекомбинации дается интегрированием выражения (20), и, таким образом, полный интегральный фактор Парселла имеет вид

$$
\begin{aligned}
F_{\rho=0}^{\mathrm{tot}, d z}= & \frac{3}{2} \int_{0}^{\pi / 2}\left|\frac{H_{m=0}^{(2)}\left(k_{\rho \mathrm{ext}} \rho_{0}\right)}{H_{m=0}^{(2)}\left(k_{\rho \mathrm{int}} \rho_{\mathrm{int}}\right)} \frac{t_{\mathrm{stTM}}^{-}}{1-r_{\mathrm{stTM}}^{+} r_{\alpha x \mathrm{TM}}\left(k_{\rho \mathrm{int}} \rho_{\mathrm{int}}\right)}\right|^{2} \\
& \times \sin ^{3}\left(\theta_{\mathrm{ext}}\right) d \theta_{\mathrm{ext}} .
\end{aligned}
$$

Если диполь находится на оси симметрии и поляризован перпендикулярно оси симметрии, то такой переход будет излучать в поперечном направлении исключительно посредством ТЕ-мод с $m= \pm 1$. Можно показать, что полный фактор Парселла для излучения в поперечном направлении для диполя, сонаправленного оси $x$ $\mathbf{d}_{f i}=\left(\mathbf{d}_{f i}\right)_{x} \mathbf{e}_{x}$ или оси $y \mathbf{d}_{f i}=\left(\mathbf{d}_{f i}\right)_{y} \mathbf{e}_{y}$ и находящегося на центральной оси, равен модовому фактору Парселла для ТЕ-моды с $m=1$.

\section{3. Результаты моделирования}

Разработанный формализм мы применяем для анализа спонтанной рекомбинации квантового дипольного перехода внутри диэлектрического цилиндра. Результаты расчета на рис. 1 показывают, что поперечное излучение дипольных переходов может как подавляться, так и усиливаться. Расстояние между пиками усиления на рис. 1 (т.е. расстояние между резонансными диаметрами цилиндра) при фиксированной поляризации моды примерно совпадает с длиной волны в материале цилиндра. Следует иметь в виду, что результаты для ТМ-моды с $m=0(m=1)$ демонстрируют полный фактор Парселла при излучении в поперечном направлении для диполя, находящегося на оси цилиндра и направленного параллельно (перпендикулярно) оси симметрии.

Можно видеть, что с ростом циклического числа $m$ максимальные значения модового фактора Парселла увеличиваются (следует обратить внимание на изменение масштаба по оси $y$ ). Особенно этот эффект выражен для первого пика, положение которого смещается в сторону бо́льших диаметров и ширина которого сужается с ростом $m$. Сужение пика и рост фактора Парселла означает появление собственной моды, добротность которой увеличивается с ростом $m$. Так как с ростом $m$ плотность энергии электромагнитного поля смещается от центра цилиндра к цилиндрической поверхности, можно сделать вывод что на рис. 1 наблюдается появление мод шепчущих галерей.

Также из рис. 1 видно, что в субволновых структурах (диаметр цилиндра много меньше длины волны) дипольные переходы, сонаправленные оси цилиндра, будут излучать в поперечном направлении намного эффективнее диполей, лежащих в поперечной плоскости. Любопытно отметить, что максимум фактора Парселла в этом случае приходится на диаметр цилиндра, примерно равный четверти длины волны в материале.

На рис. 2 рассмотрена угловая зависимость модового фактора Парселла для мод с нулевым циклическим числом $m=0$; для наглядности угол отложен от поперечного направления. Рассмотренные приведенные диаметры соответствуют пиковым значениям поперечного фактора Парселла для ТМ-мод $(D=0.26$ и 1.26) и TE-мод $(D=0.73$ и 1.73$)$. Как можно видеть на рис. 1 , максимумы поперечного фактора Парселла для ТЕ-мод примерно соответствуют минимумам для ТМ-мод и аналогично в обратную сторону.

Как видно из рис. 2, в случае диаметров, для которых поперечные факторы Парселла ТМ-мод минимальны, данные факторы увеличиваются с ростом угла и испытывают резонансное усиление рекомбинации в направлении, приближающемся к направлению оси цилиндра. Для ТЕ-мод данный эффект отсутствует, так как факторы Парселла для $D=0.26$ и 1.26 монотонно уменьшаются с ростом угла. Также можно видеть, что в цилиндрах с резонансными диаметрами для ТЕ-мод максимум фактора Парселла достигается при отклонении угла распространения ТЕ-мод от поперечного направления.

\section{4. Заключение}

Разработаны метод матриц рассеяния для расчета электромагнитного поля в среде с цилиндрической симметрией и метод квантования электромагнитного поля, основанный на матрицах рассеяния для цилиндрической среды ( $S$-квантование). Получено общее выражение для темпа спонтанной излучательной рекомбинации эмиттера в цилиндрически структурированной среде. Введены количественные характеристики, описывающие влияние цилиндрической среды на темп излучательной 
рекомбинации (модовые факторы Парселла). Получено выражение суммарного фактора Парселла для случая излучения в направлении, поперечном оси симметрии. Произведен расчет модовых факторов Парселла для диэлектрического цилиндра; продемонстрировано подавление и усиление спонтанной рекомбинации и проявление высокодобротных мод шепчущих галерей.

\section{Приложсене I}

\section{Коэффициенты отражения и прохождения для цилиндрических волн}

Коэффициенты отражения и прохождения для цилиндрической границы раздела двух диэлектриков для ТМ-волн получаются из условия непрерывности $E_{z}$ и $H_{\phi}$ компонент электромагнитного поля:

$$
\begin{aligned}
& r_{\mathrm{TM}}^{i-}=\frac{\frac{\varepsilon_{2}}{k_{\rho 2}} I_{m}^{(2)}\left(k_{\rho 2} \rho\right)-\frac{\varepsilon_{1}}{k_{\rho 1}} I_{m}^{(2)}\left(k_{\rho 1} \rho\right)}{\frac{\varepsilon_{1}}{k_{\rho 1}} I_{m}^{(1)}\left(k_{\rho 1} \rho\right)-\frac{\varepsilon_{2}}{k_{\rho 2}} I_{m}^{(2)}\left(k_{\rho 2} \rho\right)}, \\
& t_{\mathrm{TM}}^{i-}=\frac{\frac{\varepsilon_{1}}{k_{\rho 1}} I_{m}^{(1)}\left(k_{\rho 1} \rho\right)-\frac{\varepsilon_{1}}{k_{\rho 1}} I_{m}^{(2)}\left(k_{\rho 1} \rho\right)}{\frac{\varepsilon_{1}}{k_{\rho 1}} I_{m}^{(1)}\left(k_{\rho 1} \rho\right)-\frac{\varepsilon_{2}}{k_{\rho 2}} I_{m}^{(2)}\left(k_{\rho 2} \rho\right)},
\end{aligned}
$$

где введено обозначение для величины

$$
I_{m}^{(1,2)}(x)=\frac{H_{m}^{\prime(1,2)}(x)}{H_{m}^{(1,2)}(x)} \underset{x \rightarrow+\infty}{\longrightarrow} \pm i,
$$

которая в пределе большого значения аргумента стремится к плюс или минус комплексной единице.

В (П.І.1) индексом 1 обозначены параметры внешней по отношению к цилиндрической поверхности среды и индексом 2 - параметры внутренней среды; волновые векторы даются выражением $k_{\rho j}=\sqrt{\varepsilon_{j} \mu_{j} \omega_{p}^{2} / c^{2}-k_{z}^{2}}$. Для волн с нетривиальной зависимостью от циклической координаты $m \neq 0$ следует взять $k_{z}=0$ и соответственно $k_{\rho j}=K_{j}=\sqrt{\varepsilon_{j} \mu_{j}} \omega_{p} / c$. Для падения расходящейся волны из среды 2 коэффициенты имеют вид:

$$
\begin{gathered}
r_{\mathrm{TM}}^{i+}=\frac{\frac{\varepsilon_{1}}{k_{\rho 1}} I_{m}^{(1)}\left(k_{\rho 1} \rho\right)-\frac{\varepsilon_{2}}{k_{\rho 2}} I_{m}^{(1)}\left(k_{\rho 2} \rho\right)}{\frac{\varepsilon_{2}}{k_{\rho 2}} I_{m}^{(2)}\left(k_{\rho 2} \rho\right)-\frac{\varepsilon_{1}}{k_{\rho 1}} I_{m}^{(1)}\left(k_{\rho 1} \rho\right)}, \\
t_{\mathrm{TM}}^{i+}=\frac{\frac{\varepsilon_{2}}{k_{\rho 2}} I_{m}^{(2)}\left(k_{\rho 2} \rho\right)-\frac{\varepsilon_{2}}{k_{\rho 2}} I_{m}^{(1)}\left(k_{\rho 2} \rho\right)}{\frac{\varepsilon_{2}}{k_{\rho 2}} I_{m}^{(2)}\left(k_{\rho 2} \rho\right)-\frac{\varepsilon_{1}}{k_{\rho 1}} I_{m}^{(1)}\left(k_{\rho 1} \rho\right)} .
\end{gathered}
$$

Для ТЕ-мод коэффициенты определяются как отношения $E_{\phi}$ компонент электрического поля в бегущих цилиндрических волнах и вычисляются через непрерывность $E_{\phi}$ и $H_{z}$ :

$$
r_{\mathrm{TE}}^{i-}=\frac{\frac{k_{\rho 2}}{\mu_{2}} \frac{1}{I_{m}^{(2)}\left(k_{\rho 2} \rho\right)}-\frac{k_{\rho 1}}{\mu_{1}} \frac{1}{I_{m}^{(2)}\left(k_{\rho 1} \rho\right)}}{\frac{k_{\rho 1}}{\mu_{1}} \frac{1}{I_{m}^{(1)}\left(k_{\rho 1} \rho\right)}-\frac{k_{\rho 2}}{\mu_{2}} \frac{1}{I_{m}^{(2)}\left(k_{\rho 2} \rho\right)}},
$$

$$
t_{\mathrm{TE}}^{i-}=\frac{\frac{k_{\rho 1}}{\mu_{1}} \frac{1}{I_{m}^{(1)}\left(k_{\rho 1} \rho\right)}-\frac{k_{\rho 1}}{\mu_{1}} \frac{1}{I_{m}^{(2)}\left(k_{\rho 1} \rho\right)}}{\frac{k_{\rho 1}}{\mu_{1}} \frac{1}{I_{m}^{(1)}\left(k_{\rho 1} \rho\right)}-\frac{k_{\rho 2}}{\mu_{2}} \frac{1}{I_{m}^{(2)}\left(k_{\rho 2} \rho\right)}} .
$$

Для падения расходящейся TE-волны на цилиндрическую границу получим

$$
\begin{aligned}
& r_{\mathrm{TE}}^{i+}=\frac{\frac{k_{\rho 1}}{\mu_{1}} \frac{1}{I_{m}^{(1)}\left(k_{\rho 1} \rho\right)}-\frac{k_{\rho 2}}{\mu_{2}} \frac{1}{I_{m}^{(1)}\left(k_{\rho 2} \rho\right)}}{\frac{k_{\rho 2}}{\mu_{2}} \frac{1}{I_{m}^{(2)}\left(k_{\rho 2} \rho\right)}-\frac{k_{\rho 1}}{\mu_{1}} \frac{1}{I_{m}^{(1)}\left(k_{\rho 1} \rho\right)}}, \\
& t_{\mathrm{TE}}^{i+}=\frac{\frac{k_{\rho 2}}{\mu_{2}} \frac{1}{I_{m}^{(2)}\left(k_{\rho 2} \rho\right)}-\frac{k_{\rho 2}}{\mu_{2}} \frac{1}{I_{m}^{(1)}\left(k_{\rho 2} \rho\right)}}{\frac{k_{\rho 2}}{\mu_{2}} \frac{1}{I_{m}^{(2)}\left(k_{\rho 2} \rho\right)}-\frac{k_{\rho 1}}{\mu_{1}} \frac{1}{I_{m}^{(1)}\left(k_{\rho 1} \rho\right)}} .
\end{aligned}
$$

Цилиндрические волны проходят сквозь однородный цилиндрический слой без отражения. Коэффициенты прохождения от радиуса $\rho_{1}$ к радиусу $\rho_{2}$ внутри цилиндрического слоя есть

$$
\begin{aligned}
& t_{\mathrm{TM}}^{-}=\frac{H_{m}^{(2)}\left(k_{\rho} \rho_{2}\right)}{H_{m}^{(2)}\left(k_{\rho} \rho_{1}\right)}, t_{\mathrm{TM}}^{+}=\frac{H_{m}^{(1)}\left(k_{\rho} \rho_{2}\right)}{H_{m}^{(1)}\left(k_{\rho} \rho_{1}\right)}, \\
& t_{\mathrm{TE}}^{-}=\frac{H_{m}^{\prime(2)}\left(k_{\rho} \rho_{2}\right)}{H_{m}^{\prime(2)}\left(k_{\rho} \rho_{1}\right)}, t_{\mathrm{TE}}^{+}=\frac{H_{m}^{\prime(1)}\left(k_{\rho} \rho_{2}\right)}{H_{m}^{\prime(1)}\left(k_{\rho} \rho_{1}\right)} .
\end{aligned}
$$

Последовательно используя формулы для коэффициентов (П.І.1)-(П.І.6) в выражениях (6), можем получить матрицу рассеяния (5) для составной цилиндрической структуры.

\section{Приложение ІІ}

\section{Цилиндрические волны в однородной среде}

Электромагнитное поле цилиндрической ТМ-моды с циклическим числом $m$ в однородном внешнем пространстве за пределами цилиндрической структуры имеет вид:

$$
\begin{aligned}
E_{z}^{\mathrm{TMext}} & =A_{\mathrm{TM}} \frac{k_{\rho \mathrm{ext}}}{K_{\mathrm{ext}}} \\
& \times\left\{H_{m}^{(2)}\left(k_{\rho \mathrm{ext}} \rho\right)+\frac{r_{\mathrm{TM}}}{r_{\alpha x \mathrm{TM}}\left(k_{\rho \mathrm{ext}} \rho_{0}\right)} H_{m}^{(1)}\left(k_{\rho \mathrm{ext}} \rho\right)\right\}, \\
E_{\rho}^{\mathrm{TMext}} & =A_{\mathrm{TM}} \frac{k_{z}}{K_{\mathrm{ext}}} \\
\times & \left.\left\{H_{m}^{\prime(2)}\left(k_{\rho \mathrm{ext}} \rho\right)+\frac{r_{\mathrm{TM}}}{r_{\alpha x \mathrm{TM}}\left(k_{\rho \mathrm{ext}} \rho_{0}\right)} H_{m}^{\prime(1)}\left(k_{\rho \mathrm{ext}} \rho\right)\right\} .1 \mathrm{a}\right)
\end{aligned}
$$

Для ТЕ-моды поле во внешней среде будет иметь вид

$$
\begin{aligned}
E_{\phi}^{\mathrm{TEext}} & =-A_{\mathrm{TE}} i \\
& \times\left\{H_{m}^{\prime(2)}\left(k_{\rho \mathrm{ext}} \rho\right)+\frac{r_{\mathrm{TE}}}{r_{\alpha x \mathrm{TE}}\left(k_{\rho \mathrm{ext}} \rho_{0}\right)} H_{m}^{\prime(1)}\left(k_{\rho \mathrm{ext}} \rho\right)\right\},
\end{aligned}
$$




$$
\begin{aligned}
E_{\rho}^{\mathrm{TEext}} & =-A_{\mathrm{TE}} \frac{k_{\phi m}}{k_{\rho \mathrm{ext}}} \\
& \times\left\{H_{m}^{(2)}\left(k_{\rho \mathrm{ext}} \rho\right)+\frac{r_{\mathrm{TE}}}{r_{\alpha x \mathrm{TE}}\left(k_{\rho \mathrm{ext}} \rho_{0}\right)} H_{m}^{(1)}\left(k_{\rho \mathrm{ext}} \rho\right)\right\} .
\end{aligned}
$$

Коэффициенты отражения от структуры $r_{\mathrm{TM} / \mathrm{TE}}$ даются выражениями (8), а коэффициенты отражения от оси

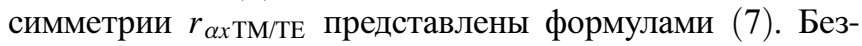
размерный профиль поля цилиндрических волн в полностью однородной среде получается из (П.ІІ.1), (П.ІІ.2) при равенстве коэффициентов отражения от структуры коэффициентам отражения от оси симметрии $r_{\mathrm{TM} / \mathrm{TE}}=r_{\alpha x \mathrm{TM} / \mathrm{TE}}$ и при $A_{\mathrm{TM} / \mathrm{TE}}=1$ :

$$
\begin{aligned}
& \tilde{E}_{z}^{\mathrm{TM} 0}=2 \frac{k_{\rho j}}{K_{j}} J_{m}\left(k_{\rho j} \rho\right), \quad \tilde{E}_{\rho}^{\mathrm{TM} 0}=2 \frac{k_{z}}{K_{j}} J_{m}^{\prime}\left(k_{\rho j} \rho\right), \\
& \tilde{E}_{\phi}^{\mathrm{TE} 0}=-2 i J_{m}^{\prime}\left(k_{\rho j} \rho\right), \quad \tilde{E}_{\rho}^{\mathrm{TE} 0}=-2 \frac{k_{\phi m}}{k_{\rho j}} J_{m}\left(k_{\rho j} \rho\right) .
\end{aligned}
$$

В векторной форме эти выражения могут быть представлены как

$$
\begin{gathered}
\tilde{\mathbf{E}}_{\mathrm{TM} 0}^{m}(\rho)=\tilde{E}_{z}^{\mathrm{TM} 0} \mathbf{e}_{z}+\tilde{E}_{\rho}^{\mathrm{TM} 0} \mathbf{e}_{\rho}, \\
\tilde{\mathbf{E}}_{\mathrm{TE} 0}^{m}(\rho)=\tilde{E}_{\phi}^{\mathrm{TE} 0} \mathbf{e}_{\phi}+\tilde{E}_{\rho}^{\mathrm{TE} 0} \mathbf{e}_{\rho} .
\end{gathered}
$$

\section{Финансирование работы}

Работа поддержана грантом Российского научного фонда (РНФ) 16-12-10503.

\section{Конфликт интересов}

Авторы заявляют, что у них нет конфликта интересов.

\section{Список литературы}

[1] J.M. Dudley, J.R. Taylor. Supercontinuum Generation in Optical Fibers (Cambridge University Press, 2010).

[2] L. Pilozzi, D. Leykam, Z. Chen, C. Conti. Optics Lett., 45, 1415 (2020).

[3] P. Yang, R. Yan, M. Fardy. Nano Lett., 10 (5), 1529 (2010).

[4] J.B. Pendry, A.J. Holden, D.J. Robbins, W.J. Stewart. J. Phys.: Condens. Matter, 10 (22), 4785 (1998).

[5] В.Г. Дубровский, Г.Э. Цырлин, В.М. Устинов. ФТП, 43 (12), 1585 (2009).

[6] M.A. Kaliteevski, S. Brand, R.A. Abram, A. Kavokin, L.S. Dang. Phys. Rev. B, 75 (23), 233309 (2007).

[7] E.I. Moiseev, N. Kryzhanovskaya, Y.S. Polubavkina, M.V. Maximov, M.M. Kulagina, Y.M. Zadiranov, A.E. Zhukov. ACS Photonics, 4 (2), 275 (2017).

[8] G.E. Cirlin, V.G. Dubrovskii, Y.B. Samsonenko, A.D. Bouravleuv, K. Durose, Y.Y. Proskuryakov, B. Mendes, L. Bowen, M.A. Kaliteevski, R.A. Abram, D. Zeze. Phys. Rev. B, 82 (3), 035302 (2010)

[9] E.M. Purcell, H.C. Torrey, R.V. Pound. Phys. Rev., 69 (1-2), 37 (1946).
[10] M.S. Tomaš. Phys. Rev. A, 51 (3), 2545 (1995).

[11] М.М. Глазов, Е.Л. Ивченко, А.Н. Поддубный, Г. Хитрова. ФTT, 53, 1665 (2011).

[12] A.R. Gubaydullin, C. Symonds, J. Bellessa, K.A. Ivanov, E.D. Kolykhalova, M.E. Sasin, A. Lemaitre, P. Scnellart, G. Pozina, M.A. Kaliteevski. Sci. Reports, 7 (1), 9014 (2017).

[13] М.А. Калитеевский, В.А. Мазлин, К.А. Иванов, А.Р. Губайдуллин. Опт. и спектр., 119 (5), 810 (2015).

Редактор А.Н. Смирнов

\section{Scattering matrix method for calculating spontaneous emission probability in cylindrically symmetrical structures}

\author{
V.V. Nikolaev ${ }^{1}$, K.A. Ivanov ${ }^{2,3}$, K.M. Morozov ${ }^{2,3}$, \\ A.V. Belonovski ${ }^{2,3}$ \\ ${ }^{1}$ loffe Institute, \\ 194021 St. Petersburg, Russia \\ 2 ITMO University, \\ 197101 St. Petersburg, Russia \\ ${ }^{3}$ St. Petersburg Academic University, \\ 194021 St. Petersburg, Russia
}

\begin{abstract}
The article develops a technique for analyzing spontaneous emission modification in structures possessing cylindrical symmetry. We develop a scattering matrix method for cylindrical structures. Based on this method we obtain a quantization method for electromagnetic field ( $S$-quantization) in cylindrical systems. General expressions for spontaneous recombination rates are obtained for an emitter placed in an arbitrary position of the structure. Quantitative values for assessment of enhancement and suppression of radiative recombination are introduced; they act as a modal Purcell factor. An expression for cumulative Purcell factor for emission in direction transversal in relation to the symmetry axis of the structure is obtained, as well as an expression for the integral Purcell factor for an emitter placed on a symmetry axis.
\end{abstract}

